\title{
BMJ Open Prospective, observational study to evaluate the relation between patient- reported outcomes and component rotation in total knee replacement: protocol for the CRACK study
}

\author{
Hans-Peter van Jonbergen (D) , ${ }^{1}$ Ellie Landman, ${ }^{1}$ Maarten Horst, ${ }^{2}$ \\ Robin Westerbeek, ${ }^{2}$ Ydo Kleinlugtenbelt ${ }^{1}$
}

To cite: van Jonbergen H-P, Landman $\mathrm{E}$, Horst $\mathrm{M}$, et al. Prospective, observational study to evaluate the relation between patient-reported outcomes and component rotation in total knee replacement: protocol for the CRACK study. BMJ Open 2019;9:e031166. doi:10.1136/ bmjopen-2019-031166

- Prepublication history for this paper is available online. To view these files, please visit the journal online (http://dx.doi. org/10.1136/bmjopen-2019031166).

Received 23 April 2019

Revised 04 September 2019

Accepted 11 October 2019

\section{Check for updates}

(c) Author(s) (or their employer(s)) 2019. Re-use permitted under CC BY-NC. No commercial re-use. See rights and permissions. Published by BMJ.

${ }^{1}$ Department of Orthopedic Surgery, Deventer Ziekenhuis, Deventer, The Netherlands

2Department of Radiology, Deventer Ziekenhuis, Deventer, The Netherlands

Correspondence to

Dr Hans-Peter van Jonbergen; vanjonbergen@dz.nl

\section{ABSTRACT}

Introduction Total knee replacement (TKR) for osteoarthritis results in a satisfactory outcome in the majority of patients, although up to one in five patients may be dissatisfied with the outcome. Persistent pain is a main contributor to patient dissatisfaction, and femoral and tibial component malrotation have been identified as a potential cause for both persistent pain and patellofemoral problems. Based on the assumption that component malrotation is the causative factor for persistent pain, early revision for patients with symptomatic malrotated components has been advocated in the literature. However, convincing evidence that component malrotation indeed results in less than optimal outcomes is lacking. This study aims to assess the relation between knee prosthesis component rotation and patient-reported outcomes in a large group of patients and to determine the range of femoral, tibial and combined rotation that results in the best clinical outcomes.

Methods and analysis In this single-centre, prospective observational cohort study, a total of 500 patients will undergo TKR. All patients will have a 3D-CT assessment of femoral and tibial component rotation within 8 weeks after surgery. Outcome measures will include the Oxford Knee Score, the Knee Injury and Osteoarthritis Outcome Score, EQ-5D, visual analogue scale for pain, the American Knee Society Score and the knee joint range of motion. We will assess the relation between femoral, tibial and combined component rotation and patient-reported outcome measures at 8 weeks and 1 year of follow-up, and we will determine the range of femoral, tibial and combined rotation that results in the best clinical outcomes.

Ethics and dissemination Ethical approval for this study has been granted by the Isala Hospital ethics committee. The results will be published in a peer-reviewed journal and presented at relevant meetings.

Trial registration number NL7635.

\section{INTRODUCTION}

Total knee replacement (TKR) for osteoarthritis results in a satisfactory outcome in the majority of patients, although up to one in five patients may be dissatisfied with the
Strengths and limitations of this study

- The prospective nature and the standardised study conditions are clear strengths of our study.

- A prospective clinical study investigating knee prosthesis component rotation and its impact on patientreported outcome measures in a large cohort of patients has never been performed.

- The findings may be highly beneficial to both practitioners and patients in deciding whether revision surgery will be beneficial.

- A limitation is that the relation between component rotation and symptoms may be implant specific.

- A further limitation is that the sample size calculation is based on the current practice of measuring component rotation only in patients with complaints.

outcome. ${ }^{12}$ One reason for reduced patient satisfaction is the presence of persistent pain, which may be associated with patient-related factors and potentially surgically modifiable mechanical causes. ${ }^{2-4}$

Knee prosthesis component malrotation has been identified as a potential cause for both persistent pain and patellofemoral joint problems. ${ }^{56}$ Two recent systematic reviews found a positive correlation between an excessive internal rotation of the components and clinical outcomes. ${ }^{78}$ However, both studies had limitations that could have affected the results, since the included studies comprised a small number of patients, did not all use patient-reported outcome measures (PROMs) and showed substantial heterogeneity in the selection of patients, the type of prosthesis used, the CT protocol and the length of follow-up.

Based on the assumption that component malrotation is the causative factor for persistent pain, early revision for patients with 
symptomatic malrotated components has been advocated in the literature. ${ }^{9}$ Some studies indeed show a benefit of revision surgery for malrotation. ${ }^{9-11}$ In marked contrast, a recent study found no relation between component rotation and pain and suggested caution when considering rotational malalignment as the cause of the pain. ${ }^{12}$

With the expected increase in both the number of primary and revision TKRs performed and the socioeconomic impact of revision surgery, ${ }^{13} 14$ it is clear that identifying a relation between component rotation and PROMs may have important consequences.

Our hypothesis is that there is a correlation between knee prosthesis component rotation and functional outcomes as assessed with PROMs. Therefore, the aim of this prospective observational study is to assess the relationship between component rotation and PROMs in a large cohort of patients and to determine the range of femoral, tibial and combined rotation that results in the best clinical outcomes.

\section{METHODS AND ANALYSIS}

\section{Study design}

This study was designed as a single-centre, prospective observational cohort study.

\section{Study population}

The study includes all mentally competent adult patients who will be treated with TKR for primary knee osteoarthritis (Kellgren and Lawrence grade III or IV). ${ }^{15}$

In order to be eligible to participate in this study, a subject must meet all of the following criteria:

- Primary knee osteoarthritis.

- Informed consent for the surgical procedure.

- Signed informed consent for the study.

A potential subject who meets any of the following criteria will be excluded from participation in this study:

- Contraindication for joint replacement surgery in general (pregnancy, active infection and severe cardiac and/or respiratory comorbidities).

- Previous distal femoral or proximal tibial fracture resulting in an altered anatomy.

- Previous osteotomies around the knee resulting in an altered anatomy.

\section{Sample size calculation}

Regression analysis will be performed to assess the relationship between the primary determinant (component rotation) and the primary study endpoint. The Oxford Knee Score (OKS) as a dichotomous outcome (improvement above the minimal clinically important difference (MCID) vs no improvement) is the primary endpoint in this analysis. In a multivariate logistic regression model, we will include possible confounders based on their effect on the beta of the primary determinant and outcome measure. Based on clinical assumption and previous literature, we would like to test five variables on confounding aspects: gender, age, body mass index (BMI), degree of degenerative changes, and predominant medial or lateral osteoarthritis.

Based on the primary analysis using logistic regression, with an alpha of 0.05 , power of $80 \%$ and $10 \%$ of patients in the unacceptable rotation group, we would need 480 patients to detect a difference of $9 \%$ in the acceptable rotation group. To account for loss to follow-up, we decided to include 500 patients in the study. Interim analysis is planned after the first 100 patients have been enrolled and have had CT assessment of component rotation. The results of this analysis will enable us to adjust the power analysis.

\section{Study parameters}

The primary endpoint of this study is the change from baseline $\mathrm{OKS}^{16}$ at 1 year of follow-up in relation to femoral, tibial and combined component rotation. Additional outcome measures include the Knee Injury and Osteoarthritis Outcome Score, ${ }^{17}$ five-level EQ-5D, visual analogue scale for pain, American Knee Society Score V.2011 18 and knee joint range of motion. In addition, we will assess the relation between rotation and the OKS as a continuous variable to obtain a more precise estimate of this relation.

\section{Study procedures}

After obtaining informed consent for the study, we will ask patients to complete the relevant PROMs. All TKRs will be performed by one of five experienced orthopaedic surgeons (with each performing $>50$ procedures per year) or residents under their direct supervision.

In all cases, a medial parapatellar arthrotomy will be used with implantation of a cemented, posterior stabilised, Persona total knee prosthesis (Zimmer-Biomet, Warsaw, Indiana). We will use the measured resection technique with anterior referencing to determine anteroposterior femoral component size. We aim for mechanical alignment. Intraoperatively, axial rotation of the femoral component will be assessed with reference to the posterior condylar line (PCL), with additional checks using the epicondylar axis and the trochlear anteroposterior axis. Tibial component rotation will be assessed using a combination of anatomical landmarks, including the tibial tuberosity, the anterior tibial crest and the anteromedial tibial contour. Coverage of the proximal tibia will be used as an additional check. The operating surgeon will specify the surgical methods used to assess femoral and tibial component rotation in the clinical records. We do not resurface the patella. Instead, circumpatellar denervation with electrocautery will be used in all patients. ${ }^{20}$

All patients will have a CT assessment of component rotation within 8 weeks after surgery. Low-dose3D-CT will be used in all cases, and measurements will be performed according to the Berger protocol. ${ }^{21}$ The angle between the surgical transepicondylar axis and the PCL of the femoral component, and between the tip of the tibial tubercle and the geometric centre of the tibial component will be assessed by two reviewers blinded to the clinical data. The 
mean of the two measurements will be used for calculation of the main study endpoint. Combined component rotation will be obtained by adding the femoral component rotational angle and the tibial component rotational angle for each knee. ${ }^{21}$

One to 3 days after surgery, patients will be discharged. Regular follow-up visits will be planned at 2 and 8 weeks and 1 year after surgery. Knee radiographs (knee anteroposterior and lateral) will be obtained at the 8-weeks and 1-year follow-up visits. At 8 weeks and 1 year after surgery, patients will be asked to complete the relevant PROMs.

\section{Data analysis}

We will present descriptive statistics for the different PROMs (preoperative, 8 weeks, 1 year postoperative and 1-year change from baseline values) and for component rotation (femoral, tibial and combined component rotation). Continuous data will be summarised using mean with SD or median with range (in case of skewed data).

The following patient demographic data will be presented in a table:

- Right or left knee (right/left).

- Sex (male/female).

- Age (in years).

- Height $(\mathrm{cm})$, weight $(\mathrm{kg})$ and BMI $\left(\mathrm{kg} / \mathrm{m}^{2}\right)$.

The following surgery-related data will be recorded:

- Number and type of previous surgeries.

- Location, degree and extent of degenerative changes.

- Size of prosthetic components.

- Additional surgical procedures performed (lateral patellar release and patellar resurfacing).

- Number and type of complications.

To assess the primary outcome, we will use an MCID of 5 points on the OKS to indicate a meaningful difference between groups. ${ }^{22}$ Logistic regression analysis will then be performed with the change from baseline OKS as the dependent (outcome) variable and the amount of component rotation as the independent variable. Furthermore, we will determine the range of femoral, tibial and combined component rotation that results in the best clinical outcomes.

\section{Patient and public involvement}

This research was done without patient involvement. Patients were not invited to comment on the study design and were not consulted to develop patient-relevant outcomes or to interpret the results. Patients were not invited to contribute to the writing or editing of this document for readability or accuracy.

\section{ETHICS AND DISSEMINATION}

The study will be conducted according to the principles of the Declaration of Helsinki (64th WMA General Assembly, Fortaleza, Brazil, October 2013) and in accordance with the Dutch Medical Research Involving Human Subjects Act (WMO) and Good Clinical Practice guidelines.
Approval for this study has been obtained from the Isala Hospital ethics committee (reference number NL68333.075.18) and the local hospital board. Following review of the protocol, the ethics committee concluded that patients will have no direct benefit from this study, and a radiation dose (approximately $0.12 \mathrm{mSv}$ ) is associated with CT.

Subjects can leave the study at any time for any reason if they wish to do so without any consequences. The investigator can decide to withdraw a subject from the study for urgent medical reasons.

The trial is registered with the Netherlands Trial Register.

The results will be published in a peer-reviewed journal and will be presented at relevant meetings. With inclusion planned in 2019 and 2020, we expect the presentation of the 1-year follow-up data in 2021.

\section{DISCUSSION}

Currently, there is no agreed consensus on the optimal strategy in patients with persistent pain following TKR. There is conflicting evidence on the role of component malrotation as a potential cause for persistent pain.

Our study is novel in that it investigates knee prosthesis component rotation in a large cohort of patients undergoing primary TKR and its impact on PROMs. This single-centre, prospective observational cohort study will help clarify whether pain and patient-reported functional outcomes are indeed related to component rotation.

Funding The authors have not declared a specific grant for this research from any funding agency in the public, commercial or not-for-profit sectors.

Competing interests None declared.

Patient consent for publication Not required.

Provenance and peer review Not commissioned; externally peer reviewed.

Open access This is an open access article distributed in accordance with the Creative Commons Attribution Non Commercial (CC BY-NC 4.0) license, which permits others to distribute, remix, adapt, build upon this work non-commercially, and license their derivative works on different terms, provided the original work is properly cited, appropriate credit is given, any changes made indicated, and the use is non-commercial. See: http://creativecommons.org/licenses/by-nc/4.0/.

ORCID iD

Hans-Peter van Jonbergen http://orcid.org/0000-0001-8722-4098

\section{REFERENCES}

1 Kahlenberg CA, Nwachukwu BU, McLawhorn AS, et al. Patient satisfaction after total knee replacement: a systematic review. Hss J 2018;14:192-201.

2 Clement ND, Bardgett M, Weir D, et al. The rate and predictors of patient satisfaction after total knee arthroplasty are influenced by the focus of the question. Bone Joint J 2018;100-B:740-8.

3 Gunaratne R, Pratt DN, Banda J, et al. Patient Dissatisfaction following total knee arthroplasty: a systematic review of the literature. $J$ Arthroplasty 2017;32:3854-60.

4 Park CN, White PB, Meftah M, et al. Diagnostic algorithm for residual pain after total knee arthroplasty. Orthopedics 2016;39:e246-52.

5 Bell SW, Young P, Drury C, et al. Component rotational alignment in unexplained painful primary total knee arthroplasty. Knee 2014;21:272-7. 
6 Bhattee G, Moonot P, Govindaswamy R, et al. Does malrotation of components correlate with patient dissatisfaction following secondary Patellar resurfacing? Knee 2014;21:247-51.

7 Valkering KP, Breugem SJ, van den Bekerom MPJ, et al. Effect of rotational alignment on outcome of total knee arthroplasty. Acta Orthop 2015;86:432-9.

8 Panni AS, Ascione F, Rossini M, et al. Tibial internal rotation negatively affects clinical outcomes in total knee arthroplasty: a systematic review. Knee Surg Sports Traumatol Arthrosc 2018;26:1636-44.

9 Lakstein D, Zarrabian M, Kosashvili Y, et al. Revision total knee arthroplasty for component malrotation is highly beneficial: a case control study. J Arthroplasty 2010;25:1047-52.

10 Sternheim A, Lochab J, Drexler M, et al. The benefit of revision knee arthroplasty for component malrotation after primary total knee replacement. Int Orthop 2012;36:2473-8.

11 Pietsch M, Hofmann S. Early revision for isolated internal malrotation of the femoral component in total knee arthroplasty. Knee Surg Sports Traumatol Arthrosc 2012;20:1057-63.

12 Young SW, Saffi M, Spangehl MJ, et al. Unexplained pain following total knee arthroplasty: is rotational malalignment the problem? Knee 2018;25:329-34.

13 Kurtz S, Ong K, Lau E, et al. Projections of primary and revision hip and knee arthroplasty in the United States from 2005 to 2030. J Bone Joint Surg Am 2007;89:780-5.
14 Kurtz SM, Ong KL, Schmier J, et al. Future clinical and economic impact of revision total hip and knee arthroplasty. J Bone Joint Surg Am 2007;89 Suppl 3:144-51.

15 Kellgren JH, Lawrence JS. Radiological assessment of OsteoArthrosis. Ann Rheum Dis 1957;16:494-502.

16 Haverkamp D, Breugem SJM, Sierevelt IN, et al. Translation and validation of the Dutch version of the Oxford 12-Item knee questionnaire for knee arthroplasty. Acta Orthop 2005;76:347-52.

17 de Groot IB, Favejee MM, Reijman M, et al. The Dutch version of the knee injury and osteoarthritis outcome score: a validation study. Health Qual Life Outcomes 2008;6:16.

18 Scuderi GR, Bourne RB, Noble PC, et al. The new knee Society knee scoring system. Clin Orthop Relat Res 2012;470:3-19.

19 Van Der Straeten C, Witvrouw E, Willems T, et al. Translation and validation of the Dutch new knee Society scoring system. Clin Orthop Relat Res 2013;471:3565-71.

20 van Jonbergen HPW, Scholtes VAB, Poolman RW. A randomised, controlled trial of circumpatellar electrocautery in total knee replacement without Patellar resurfacing: a Concise follow-up at a mean of 3.7 years. Bone Joint J 2014;96-B:473-8.

21 Berger RA, Crossett LS, Jacobs JJ, et al. Malrotation causing patellofemoral complications after total knee arthroplasty. Clin Orthop Relat Res 1998;356:144-53.

22 Clement ND, MacDonald D, Simpson AHRW. The minimal clinically important difference in the Oxford knee score and short form 12 score after total knee arthroplasty. Knee Surg Sports Traumatol Arthrosc 2014;22:1933-9. 